Pacific

Journal of

Mathematics

CLEANLINESS OF GEODESICS IN HYPERBOLIC 3-MANIFOLDS

C. Adams, A. Colestock, J. Fowler, W. Gillam, and E.

KATERMAN 


\title{
CLEANLINESS OF GEODESICS IN HYPERBOLIC 3-MANIFOLDS
}

\author{
C. Adams, A. Colestock, J. Fowler, W. Gillam, and E. \\ KATERMAN
}

In this paper, we investigate geodesics in cusped hyperbolic 3-manifolds. We derive conditions guaranteeing the existence of geodesics avoiding the cusps and use these geodesics to show that in "almost all" finite volume hyperbolic 3-manifolds, infinitely many horoballs in the universal cover corresponding to a cusp are visible in a fundamental domain of the cusp when viewed from infinity.

\section{Introduction.}

Let $M$ be a cusped hyperbolic manifold of finite volume. Lift $M$ to the upper-half space model of $\mathbb{H}^{3}$, so that cusps lift to horoballs. Center one of the horoballs at infinity, then expand one cusp until it just touches itself or another cusp. Inflate each cusp in turn until all have maximal size without intersecting themselves or another cusp. The result is a maximal cusp or maximal set of cusps. Looking down from infinity, we see a pattern of horoballs within the fundamental parallelogram for the cusp subgroup that fixes $\infty$. Call the set of horoballs in the parallelogram the horoball diagram for the manifold. If the horoballs are opaque, how many are visible? Do finitely many horoballs suffice to cover the fundamental parallelogram? This question, first asked by Darren Long, is answered below.

There are cusped hyperbolic 3-manifolds in which a viewer situated at infinity can see only finitely many horoballs in the horoball diagram. This is true of the figure-eight knot complement because the first three layers of horoballs cover the plane (see Figure 1). Since this is not obvious from a diagram, we prove this fact in Section 4. And, because any singly cusped finite cover of the figure-eight knot complement will also have this property, there are infinitely many examples of manifolds with this property. But remarkably, such examples are exceptional:

Theorem 1.1. In the horoball diagram of almost all finite volume hyperbolic 3-manifolds, infinitely many horoballs are visible from infinity. 


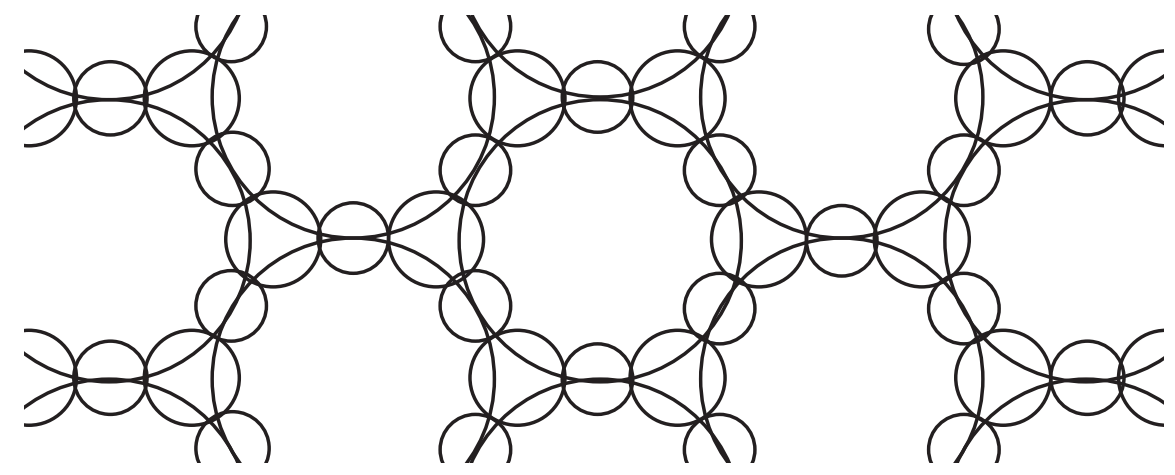

Figure 1. For the figure-eight knot, the plane is covered by the top three layers of horoballs.

In Section 2, we define "almost all" and discuss the notation used throughout the paper. In Section 3, we prove Theorem 1.1. In Section 4, we investigate the properties of the figure-eight knot complement and the Whitehead link complement. Finally, we explain various implications of these results in Section 5 by relating our ideas to number theoretic problems. We are grateful to Ian Agol for bringing these questions to our attention.

\section{Preliminaries.}

Here we list the conventions used in this paper. We will be working in the upper-half plane model of $\mathbb{H}^{2}$ and the upper-half space model of $\mathbb{H}^{3}$, and points will be denoted $p=(z, t) \in \mathbb{H}^{3}$ (or $p=(x, t) \in \mathbb{H}^{2}$ ) where $x, t \in \mathbb{R}, z \in \mathbb{C}$ and $t>0$; that is, $\partial \mathbb{H}^{3}=\mathbb{C} \cup\{\infty\}$ and $\partial \mathbb{H}^{2}=\mathbb{R} \cup\{\infty\}$. Given two points $p_{1}, p_{2} \in \mathbb{H}^{n}$, the Euclidean length between $p_{1}$ and $p_{2}$ will be denoted $\ell_{e}\left(p_{1}, p_{2}\right)$. The hyperbolic length between points $p_{1}$ and $p_{2}$ will be denoted $\ell\left(p_{1}, p_{2}\right)$. A cusp in a hyperbolic 3 -manifold is a subset with interior homeomorphic to $T^{2} \times(0,1)$ which lifts to a set of horoballs in $\mathbb{H}^{3}$. Choosing one such horoball to be centered at $\infty$, the cusp subgroup is the $\mathbb{Z}+\mathbb{Z}$ subgroup of the fundamental group of the manifold realized as a discrete group of fixed point free isometries. A fundamental domain for the action of the cusp subgroup on the boundary plane $\mathbb{C}$ is a parallelogram $P$. Horocycles or horodiscs in $\mathbb{H}^{2}$ will be denoted by $h$ or $h_{i}$, and horospheres or horoballs in $\mathbb{H}^{3}$ by $H$ and $H_{i}$. In both cases, the corresponding Euclidean radii will be denoted by $r, r_{i}, r, r_{i} \in \mathbb{R}^{+}$respectively.

Definition 2.1. We say that almost all finite volume hyperbolic 3-manifolds have a property $Q$ if, for any real number $V>0$, all but finitely many with volume less than $V$ have property $Q$.

Stated differently, "almost all" is "all but a finite number below any given volume." 
Definition 2.2. A geodesic is singly orthogonal if it intersects the cusp boundary exactly once and does so at a right angle; a doubly orthogonal geodesic intersects the cusp boundary twice perpendicularly.

That is, a singly orthogonal geodesic is perpendicular to the tangent plane of the cusp at the point of intersection. Note that a geodesic entering the cusp perpendicularly stays in the cusp from then on.

A cusped hyperbolic 3-manifold has infinitely many doubly orthogonal geodesics connecting any cusp to itself and infinitely many connecting any cusp to any other cusp. We can see this by lifting cusps to horoballs, centering a horoball at infinity, and then we find infinitely many doubly orthogonal geodesics by drawing the vertical geodesics connecting the centers of horoballs and infinity. If we are allowed to alter the size of the cusps, we shall choose a configuration such that no cusp overlaps the interior of itself or another cusp. Below, we will use $\ell_{h}$ to denote the hyperbolic length of a doubly orthogonal geodesic outside the cusp or cusps. Usually, the size of the cusps is chosen to maximize cusp volume, and in the case of a 1-cusped hyperbolic 3-manifold, this choice is canonical. Given any geodesic in a cusped hyperbolic 3-manifold, we have the following definition:

Definition 2.3. A dirty geodesic intersects the cusp boundary non-orthogonally at least once, a spotted geodesic intersects the cusp boundary tangentially at least once and perpendicularly otherwise, and a clean geodesic is neither dirty nor spotted.

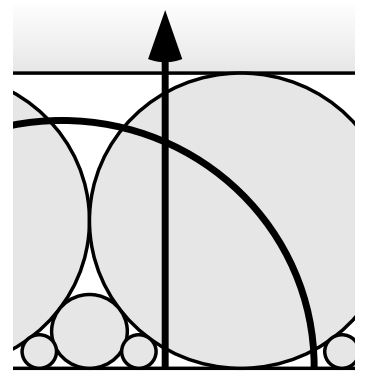

(a) Dirty

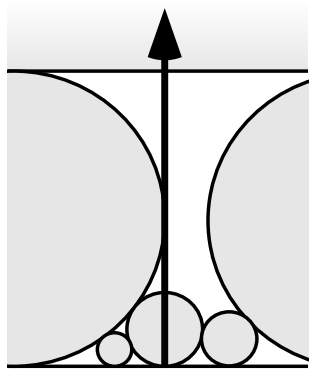

(b) Spotted

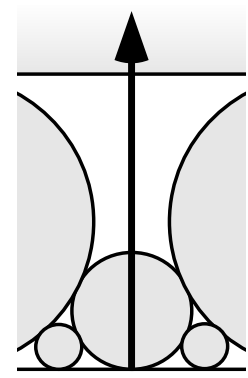

(c) Clean

Figure 2. The various possibilities for a geodesic intersecting the cusp.

For examples of lifts of geodesics of each type see Figure 2. We will find that the existence of geodesics of these various types is intimately related to the question of horoball visibility. 


\section{Horoball visibility and cleanliness.}

We want to show that for almost all hyperbolic 3-manifolds, infinitely many horoballs are visible. The proof consists of three parts. First, we show that infinitely many horoballs are visible if there is a clean singly orthogonal geodesic. Second, there is a clean singly orthogonal geodesic if there is a "short" closed geodesics. Third, there are "short" closed geodesics in almost all hyperbolic 3-manifolds.

Theorem 3.1. If a hyperbolic 3-manifold $M$ contains a clean singly orthogonal geodesic then infinitely many horoballs in the horoball diagram are visible from $\infty$.

Proof. Suppose that $M$ contains a clean singly orthogonal geodesic $\alpha$. Let $P$ be a parallelogram in $\mathbb{C}$ that is a fundamental domain for the cusp subgroup and let $\widetilde{\alpha}$ be a lift of $\alpha$ to $\mathbb{H}^{3}$ with one endpoint at infinity and the other endpoint in $P$. Suppose that only finitely many horoballs are visible. Then there is some $\epsilon>0$ such that all horoballs $H_{i}^{*}$ with radius $r_{i}^{*}<\epsilon$ are in the shadows of the horoballs $H_{i}$ of radius $r_{i} \geq \epsilon$. Then there is a neighborhood of $\widetilde{\alpha}$ of Euclidean radius $\delta>0$ which is disjoint from all the $H_{i}$. Since horoball centers are dense in $\mathbb{C}$, there must be a horoball $H$ whose center lies in this neighborhood, and consequently $H$ has radius $r<\epsilon$. Since part of $H$ lies in the $\delta$-neighborhood of $\widetilde{\alpha}$, the projection of $H$ to $\mathbb{C}$ is not completely covered by the projections of the $H_{i}$.

Although the ability to see infinitely many horoballs from infinity may not imply the existence of a clean singly orthogonal geodesic, there is a partial converse to the above result.

Theorem 3.2. Given a hyperbolic 3-manifold $M$, if infinitely many horoballs in the horoball diagram are visible from $\infty$, then $M$ contains a clean or spotted singly orthogonal geodesic.

Proof. The fundamental parallelogram $P$ for the cusp subgroup of $M$ is a compact subset of $\mathbb{C}$. Let $H_{0}$ denote one of the largest horoballs with center in $P$. Choose $H_{i+1}$ to be one of the largest horoballs with center in $P$ which is smaller than $H_{i}$ and whose projection to $\mathbb{C}$ is not completely covered by the projections of larger horoballs. $H_{i}$ exists for all $i$ by assumption. Let $c_{i}$ denote the center of $H_{i}$; since $P$ is compact, we can pick $a \in P$ a limit point of $\left\{c_{i}\right\}$. Then $a$ cannot lie in the interior of the projection of a horoball $H$ because if it did, then we could choose an $N$ large enough so that $H_{N}$ is also completely contained within the projection of $H$. So, either $a$ is outside the projections of all horoballs or $a$ lies on the boundary of the projection(s) of one or more horoballs. We conclude that the geodesic from $a$ to $\infty$ covers a clean singly orthogonal or spotted singly orthogonal geodesic in the manifold. 
Corollary 3.3. If a hyperbolic 3-manifold $M$ contains infinitely many clean doubly orthogonal geodesics then $M$ contains a clean or spotted singly orthogonal geodesic.

Proof. If $M$ contains infinitely many clean doubly orthogonal geodesics, then the fundamental parallelogram for $M$ in $\mathbb{H}^{3}$ contains infinitely many horoballs such that the geodesic from the top of each horoball to the horoball at infinity does not touch any other horoballs. Since the projection of each such horoball to $\mathbb{C}$ contains a region uncovered by the other horoballs, the result follows immediately from Theorem 3.2.

There are many relationships between geodesic length and clean geodesics. For example, any doubly orthogonal geodesic $g$ of hyperbolic length $\ell_{h}(g)<$ $\log 4$ is clean. Some geometry shows that there is not enough room for a third horoball to fit between the two horoballs pierced by the geodesic. (see Figure 3).

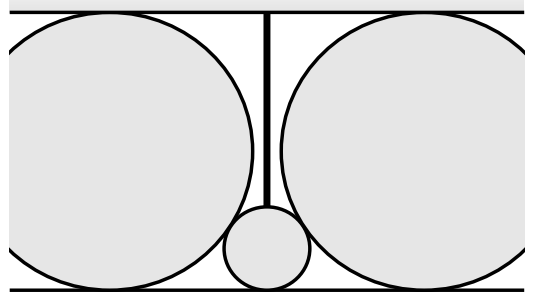

Figure 3. If a doubly orthogonal geodesic is short enough, then no horoball can intersect it.

We also look at simple closed geodesics, and define them to be clean if they avoid the cusps.

Lemma 3.4. Any closed geodesic g of hyperbolic length $\ell_{h}(g)<\log (2+2 \sqrt{2})$ is clean.

Proof. We must prove that if $g$ is short enough, then there is a tubular neighborhood around $g$ that misses all horoballs. Let $g$ be the closed geodesic lifting to a vertical geodesic $\widetilde{g}$, so that $g$ has endpoints $(0,1)$ and $\left(0,1+\ell_{e} g\right)$ and $\widetilde{g}$ has ideal endpoints $(0,0)$ and $\infty$. Let $H_{1}$ denote the horoball centered at one endpoint of the semi-circular geodesic which intersects $\widetilde{g}$ at $(0,1)$, and let $H_{2}$ be the horoball centered a Euclidean distance $\ell_{e}(g)+1$ from the origin on the same line as the origin and the center of $H_{1}$. We want to find $g$ so that when we inflate both $H_{1}$ and $H_{2}$, then the moment they become tangent to each other will be the exact moment when they each become tangent to $g^{*}$. We know that $r_{1}=1, r_{2}=\ell_{e}(g)+1$, and the distance between the centers of $H_{1}$ and $H_{2}$ is $d\left(H_{1}, H_{2}\right)=\ell_{e}(g)$. Thus, from the triangle whose 
hypoteneuse is the line between the Euclidean centers of $H_{1}$ and $H_{2}$, we find that $\ell_{e}(g)=2+2 \sqrt{2}$.

Surprisingly, if a manifold has a sufficiently short closed geodesic, there is a clean singly orthogonal geodesic. Before we prove the theorem for 3manifolds, we will first prove the corresponding result for 2-manifolds.

Lemma 3.5. A cusped hyperbolic 2-manifold with a closed geodesic $g$ of hyperbolic length $\ell_{h}(g)<\log 3$ will have a clean singly orthogonal geodesic.

Proof. Lift the hyperbolic 2-manifold to the upper half-plane model, lifting $g$ to a vertical geodesic $\widetilde{g}$. Expand a tubular neighborhood about $\widetilde{g}$ until it just touches the cusp, say at a horocycle $h$. Draw the vertical geodesic $\widetilde{g}^{\prime}$ heading up from the center of $h$ and leaving the cusp orthogonally. Intuitively, if the tubular neighborhood is wide enough (i.e., the geodesic is short enough), then there will not be enough room above $h$ and below the tubular neighborhood for another horocycle to hit $\widetilde{g}^{\prime}$, which will make $\widetilde{g}^{\prime}$ a clean singly orthogonal geodesic. See Figure 4.

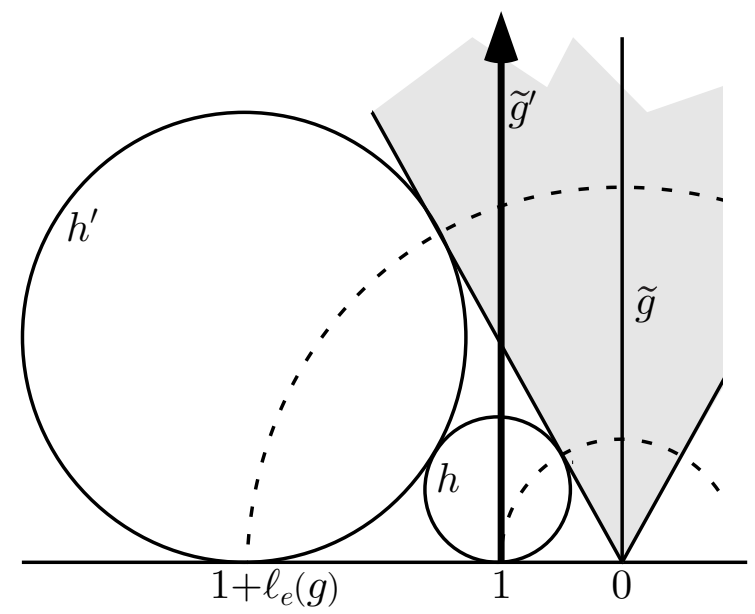

Figure 4. A short geodesic with a wide neighborhood.

Suppose $h$ has a Euclidean radius of $r$, and let $h^{\prime}$ be the image of $h$ under the isometry generating the closed geodesic $g$, so $h^{\prime}$ is a horocycle of radius $\left(1+\ell_{e}(g)\right) r$. For convenience, choose coordinates so that the vertical line $g$ sits at 0 , and the horocycle $h$ has center at 1 , so $h^{\prime}$ has center at $\left(1+\ell_{e}(g)\right)$. Now $r$ is as large as possible when $h$ and $h^{\prime}$ are tangent, which gives

$$
r \leq \sqrt{\frac{\ell_{e}(g)^{2}}{4+4 \ell_{e}(g)}} .
$$


But if

$$
r\left(1+\ell_{e}(g)\right) \leq \ell_{e}(g),
$$

then $h^{\prime}$ doesn't hit $\widetilde{g}^{\prime}$. And if $h^{\prime}$ doesn't hit $\widetilde{g}^{\prime}$, then no horocycle can hit $\widetilde{g}^{\prime}$, because $h^{\prime}$ was chosen to be maximal. Putting inequalities (3.1) and (3.2) together gives us the sufficient condition that $\ell_{e}(g)<3$ (or equivalently that $r<3 / 4)$.

The proof for the 3-manifold case is similar, except that we have to take into account the loxodromic nature of a closed geodesic in $\mathbb{H}^{3}$. We utilize ideas similar to those appearing in [Mey86].

Theorem 3.6. Any cusped hyperbolic 3-manifold with a closed geodesic of length less than 0.1777 will have a clean singly orthogonal geodesic.

Proof. Lift the manifold to the upper half-space model in such a way that the given closed geodesic lifts to a vertical geodesic above the origin. Expand a tubular neighborhood of this closed geodesic until it just hits the cusp. Let $H$ be a horoball tangent to the neighborhood, and suppose the center of $H$ is at $(1,0)$ and has radius $r$. Now let $\widetilde{g}^{\prime}$ be the vertical geodesic between $(0,1)$ and $\infty$; we claim that $\widetilde{g}^{\prime}$ is the desired singly orthogonal clean geodesic.

Suppose a horoball hits $\widetilde{g}^{\prime}$. We rotate this horoball around $\widetilde{g}^{\prime}$ until the center of the horoball is at some positive real number. This situation is equivalent to the $\mathbb{H}^{2}$ case, and by the previous lemma, we know that another horoball cannot hit $\widetilde{g}^{\prime}$ provided $r<3 / 4$.

We therefore want to bound $r$ based on $g$. We assume that $\theta$, the twist of $g$, is less than $2 \pi / 6$. Let $H^{\prime}$ be the image of $H$ under the isometry corresponding to the closed geodesic $g$. The radius $r$ is maximized when $H$ and $H^{\prime}$ are tangent. By the law of cosines, we bound the radius:

$$
r \leq \sqrt{\frac{1+\left(1+\ell_{e}(g)\right)^{2}-2\left(1+\ell_{e}(g)\right) \cos \theta}{4+4 \ell_{e}(g)}} .
$$

Assuming $-2 \pi / 6<\theta<2 \pi / 6$, we have $r<3 / 4$ whenever $\ell_{e}(g)<(13+$ $\sqrt{105}) / 8$, which we will denote by $k$. Thus, if the hyperbolic length of $g$ is less than $\log k$ and the twist of $g$ is less than $2 \pi / 6$, then we have our singly orthogonal clean geodesic.

But by unwinding $g$ up to six times, we can always get a closed geodesic with twist less than $2 \pi / 6$. So if we have a closed geodesic with any twist and of hyperbolic length less than $(\log k) / 6 \approx 0.1777$, then we will have a singly orthogonal clean geodesic.

It's important to realize that our assumption of $\theta<2 \pi / 6$ was not arbitrary; choosing $2 \pi / 6$ produces the best possible bound with this method.

Lemma 3.7. Given a real number $\epsilon>0$, almost all hyperbolic 3-manifolds have a simple closed geodesic of length less than $\epsilon$. 
Proof. Pick a value $V>0$. By the results of Thurston and Jørgensen (cf. [Thu79]), every hyperbolic 3-manifold of volume less than $V$ comes from surgery on a finite set of cusped hyperbolic 3-manifolds. Therefore, for any given bound $B$, all but finitely many of the manifolds of volume less than $V$ will have a pair of surgery coefficients $(p, q)$ that satisfy $p^{2}+q^{2}>B$. By choosing $B$ large, we can insure that the core of the surgery becomes a simple closed geodesic of length less than $\epsilon$.

The following theorem completes the proof of the main result of this paper:

Theorem 3.8. In almost all hyperbolic 3-manifolds, infinitely many horoballs are visible looking down from infinity to the fundamental parallelogram.

Proof. By the previous lemma, almost all hyperbolic 3-manifolds have a geodesic $g$ where $\ell_{h}(g)<0.17$. But by Theorem 3.6, a hyperbolic 3 -manifold with a closed geodesic shorter than 0.17 has a clean singly orthogonal geodesic. And by Theorem 3.1, a clean singly orthogonal geodesic means that infinitely many horoballs are visible.

\section{Examples.}

An example of a manifold that does not have a clean singly orthogonal geodesic is the figure-eight knot complement.

Lemma 4.1. The first three layers of horoballs that make up the horoball packing of the figure-eight knot complement project to cover $\mathbb{C}$ entirely.

Proof. Let $H_{i}$ denote the $i^{\text {th }}$ largest horoball in the cusp diagram of $K$ for $1 \leq i \leq 3$. Let $r_{i}$ be the radius of $H_{i}$ and let $d\left(H_{i}, H_{j}\right)$ be the distance between the centers of tangent horoballs $H_{i}$ and $H_{j}$ where the distance between the centers of $H_{i}$ and its nearest copy is computed if $i=j$. Since certain copies of $H_{1}, H_{2}$, and $H_{3}$ are pairwise tangent, we can find $d\left(H_{i}, H_{j}\right)$ for $1 \leq i \leq 3,1 \leq j \leq 3$. Since $H_{1}$ and its nearest translate are tangent to one another and to the horoball centered at infinity, we see that $d\left(H_{1}, H_{1}\right)=$ 1. Next, we can find $d\left(H_{1}, H_{2}\right)$ by noting that the hexagonal packing of the copies of $H_{1}$ force the centers of the copies of $H_{2}$ to be in the center of the equilateral triangle with vertices at the centers of the copies of $H_{1}$. Since $r_{1}=1 / 2$, we find that

$$
d\left(H_{1}, H_{2}\right)=\frac{1}{2} \frac{1}{\sin \frac{\pi}{6}}=\frac{1}{\sqrt{3}} .
$$

It is not hard to see that $\left(1 / 2+r_{2}\right)^{2}=\left(1 / 2-r_{2}\right)^{2}+1 / 3$ and therefore that $r_{2}=1 / 6$. Similarly, since $d\left(H_{1}, H_{3}\right)=1 / 2$, we find that $r_{3}=1 / 8$. 
Let $D_{i}$ denote the projection of the interior of $H_{i}$. Now, to show that the copies of $D_{1}, D_{2}$, and $D_{3}$ completely cover $\mathbb{C}$, it is sufficient to show that

$$
\bigcap_{i=1}^{3} D_{i} \neq \emptyset \text {. }
$$

And, to show this, it suffices to show that $\theta_{1}+\theta_{2}>\pi / 6$, where $\theta_{1}$ is the angle between the line segment joining the centers of $H_{1}$ and $H_{2}$ and the line segment joining the center of $H_{1}$ with the point where $\partial D_{1}$ and $\partial D_{2}$ intersect, and $\theta_{2}$ is similarly defined for $H_{1}$ and $H_{3}$. Now we have

$$
\frac{1}{36}=\frac{1}{3}+\frac{1}{4}-2\left(\frac{1}{\sqrt{3}}\right)\left(\frac{1}{2}\right) \cos \theta_{1} \text {. }
$$

And thus, $\theta_{1}=\cos ^{-1}\left(\frac{5 \sqrt{3}}{9}\right)$, and similarly $\theta_{2}=\cos ^{-1}\left(\frac{31}{32}\right)$. (For helpful pictures, see Figure 1 or refer to SnapPea [Wee].)

Corollary 4.2. In the figure-eight knot complement:

(1) There are no clean singly orthogonal geodesics.

(2) There are only finitely many doubly orthogonal clean geodesics.

Proof. The first part follows immediately from the above theorem. An infinitely long clean geodesic can be pictured as a vertical geodesic leaving the horoball centered at $\infty$, but all vertical geodesics in the figure-eight knot complement must intersect the cusp a second time by the theorem.

The second part also follows immediately since there are only finitely many copies of the largest three horoballs in the fundamental domain for the cusp of the figure-eight knot complement in the universal cover.

An example of a manifold that has a horoball pattern which does not completely cover $\mathbb{C}$ and thus from which infinitely many horoballs are visible from infinity is the Whitehead link complement.

Lemma 4.3. The Whitehead link complement considered with symmetric tangent cusps has a clean singly orthogonal geodesic.

Proof. Take the set of horoballs in the upper-half-space model with centers at $\frac{p}{q}$ (reduced) where $p, q \in \mathbb{Q}(\sqrt{(-1)})$ and diameters given by $\frac{1}{k q \bar{q}}$. The horoball centered at $\{\infty\}$ is respresented by a horizontal plane at height $\frac{k}{2}$. This set of horoballs is invariant under the Picard group and therefore projects to the two cusps in the Whitehead link complement. Ford [For25] demonstrated that in such a space there exists a semi-circle $C$ with endpoints $\left(\frac{1}{2},-\frac{\sqrt{3}}{2}, 0\right)$ and $\left(\frac{1}{2}, \frac{\sqrt{3}}{2}, 0\right)$ and radius $\frac{\sqrt{3}}{2}$, that is tangent to an infinite number of horoballs without entering the interior of any when $k=\sqrt{3}$. Although these horoballs are not disjoint, we can shrink back the resultant cusps in the Whitehead link at a symmetric rate until the horoball centered at infinity has boundary equal to the horizontal plane at height 1 , at which point 
all of the horoballs have disjoint interiors. Then there is a tube, $t$, of radius $\log \frac{2}{\sqrt{3}}$ around $C$ that does not intersect any horoballs in its interior. Let $v$ be a singly orthogonal geodesic with basepoint $\left(\frac{1}{2}, \frac{\sqrt{3}}{2}\right)$. Moving upwards towards $\infty, v$ travels within $t$ until the point $s=\left(\frac{1}{2}, \frac{\sqrt{3}}{2}, \frac{1}{4}\right)$ and thus must be clean within that region. Above $s$, the only balls that might intersect $v$ are those with diameter at least $\frac{1}{4}$. But by looking at the balls centered at $\frac{1+i}{2+i}, \frac{0+i}{1+2 i}$, and $\frac{1+0 i}{2+0 i}$, we see that they are mutually tangent and enclose an area around $v$ which does not cover it's basepoint and prevents any balls of diameter at least $\frac{1}{4}$ from being in that region. Thus $v$ is a clean singly orthogonal geodesic.

Theorem 4.4. Infinitely many horoballs are visible from infinity within the fundamental parallelogram of the Whitehead link complement when considered with symmetric tangent cusps.

Proof. This follows immediately from the above lemma and Theorem 3.1.

\section{Number theory.}

Many of these questions have relevance to number theory. In every cusped hyperbolic 3-manifold, the centers of the horoballs form a subfield of the complex plane. For example, if $\Gamma$ is a torsion-free subgroup of finite index in a Bianchi group, then $\mathbb{H}^{3} / \Gamma$ is a finite volume hyperbolic 3-manifold and questions about clean singly orthogonal geodesics are closely related to standard diophantine approximation.

Let $\alpha$ be a real irrational number. In 1891, Hurwitz showed that the inequality

$$
\left|\alpha-\frac{p}{q}\right|<\frac{1}{k|q|^{2}}
$$

has infinitely many solutions in coprime integers $p$ and $q$ when $k=\sqrt{5}$, and that $\sqrt{5}$ is the best constant possible. This inequality can be generalized to approximate complex numbers.

Let $d$ be a positive square-free integer and let $O_{d}$ be the ring of integers in $\mathbb{Q}(\sqrt{-d})$. Let $\alpha \in \mathbb{C}-\mathbb{Q}(\sqrt{-d})$. Denote by $k_{d}(\alpha)$ the supremum of all $k$ such that the inequality above has infinitely many solutions in $p, q \in O_{d}$. Define the set of numbers

$$
L_{d}=\left\{1 / k_{d}(\alpha): \alpha \in \mathbb{C}-\mathbb{Q}(\sqrt{-d})\right\}
$$

to be the Lagrange spectrum for the imaginary quadratic number field $\mathbb{Q}(\sqrt{-d})$ and $C_{d}=\sup L_{d}$ the Hurwitz constant for the field. See [For25] and $[\mathbf{V u l}]$.

Geometrically, this inequality indicates that if we expand the horoballs for a given manifold, $M=\mathbb{H}^{3} / \Gamma$ where $\Gamma$ is a torsion-free subgroup of finite 
index in a Bianchi group, until they all have radii equal to $C_{d} \backslash|q|^{2}$ then any vertical geodesic with basepoint $\alpha$ will intersect an infinite number of horoballs. But horoballs with such radii are not necessarily disjoint. An interesting question which presents itself is, what happens if we allow the radii of the horoballs to correspond to those of a maximal cusp, thereby ensuring that the horoballs are disjoint? This is equivalent to studying the inequality when $k=1$.

As we have shown, the plane is completely covered by the horoballs in a maximal cusp of the figure eight knot complement. Therefore, there exists at least one solution to the inequality for all $\alpha$ 's when $k=1$. With the Whitehead link complement, however, the existence of a clean singly orthogonal geodesic implies that if $\alpha=\frac{1}{2}+\frac{\sqrt{3}}{2} i$ then the inequality has no solutions. These questions could be applied to other hyperbolic 3-manifolds besides those associated with the Bianchi groups.

\section{References}

[For25] L. Ford, On the closeness of approach of complex rational fractions to a complex irrational number, Trans. Amer. Math. Soc., 2 (1925), 146-154, MR 1501304, Zbl 51.0157.03.

[Mey86] R. Meyerhoff, A lower bound for the volume of hyperbolic 3-manifolds, Can. J. Math., XXXIX (1987), 1038-1056, MR 88k:57049, Zbl 0694.57005.

[Thu79] W. Thurston, The Geometry and Topology of Three-Manifolds, Notes, Dept. of Math., Princeton University, 1979. See also Three-Dimensional Geometry and Topology. Vol. 1, Princeton Mathematical Series, 35, Princeton University Press, Princeton, NJ, 1997, MR 1435975, Zbl 0873.57001.

[Vul] L.Ya. Vulakh, Diophantine approximation on Bianchi groups, J. Number Theory, 54 (1995), 73-80, MR 96g:11076, Zbl 0838.11027.

[Wee] J. Weeks, SnapPea, A computer program for creating and studying hyperbolic 3-manifolds, available at http://www.geometrygames.org/SnapPea/index.html.

Received October 1, 2002 and revised January 20, 2003. This research was supported by Williams College and the National Science Foundation under grants DMS-9820570 and DMS-9803362. 
E-mail address: Colin.Adams@williams.edu

Dept. OF Mathematics

Williams College

Williamstown, MA 01267

Dept. OF MATHEMATiCs

Williams College

Williamstown, MA 01267

Dept. OF MATHEMATiCs

Williams College

Williamstown, MA 01267

DePt. of Mathematics

Williams College

Williamstown, MA 01267 